\title{
Municipio e igualdad de género en México: un análisis institucional
}

\section{Municipality and gender equality in Mexico: an institutional analysis}

AUTORES: José Luis Salazar Martínez Alberto Arellano Ríos

SUMARIO: I. Introducción. II. La dimensión del problema: a manera de contextualización. III. La igualdad de genero en el marco institucional. IV. Políticas públicas locales con perspectiva de género. V. A manera de cierre.

Resumen: En este documento se delinea el marco institucional y los mecanismos que le permiten al municipio mexicano diseñar e implementar políticas públicas con perspectivas de género. Para la consecución de dicho objetivo se contextualiza la situación de desigualdad en México. Posteriormente se traza el marco normativo imperante y delinean algunos elementos metodológicos para el diseño, implementación y evaluación de las políticas de igualdad de género en el municipio.

Palabras clave: municipio mexicano, igualdad de género, marco jurídico, políticas públicas

Abstract: This paper describes the institutional framework and mechanisms that allow the Mexican municipality to design and implement public policies with outlined gender perspectives. To achieve this objective the inequality in Mexico is contextualized. Subsequently the prevailing regulatory framework is outlined with some methodological trace elements for its design, implementation and evaluation of gender equality policies in the municipality.

Keywords: Mexican municipality, gender equality, legal framework, public policies

\section{Introducción ${ }^{1}$}

¿Cuál es el marco institucional y los mecanismos que le permiten al municipio mexicano diseñar e implementar políticas públicas con perspectivas de género? Es la pregunta que articula este ensayo académico en el cual no sólo se traza el diseño formal e institucional que los gobiernos locales tienen para coadyuvar a tal cometido, sino que se justiprecian los alcances y limitaciones de tales instrumentos. Para la consecución de tal fin el documento se divide en tres apartados, en el primero de ellos se hace notar la dimensión

\footnotetext{
1 Parte del contenido de este documento formó parte del proyecto denominado "desarrollo local con igualdad de género" que se elaboró para el Instituto Nacional de las Mujeres en 2009. El objetivo principal fue proporcionar un manual a las autoridades municipales mexicanas acerca del marco institucional y del enfoque de políticas públicas para el desarrollo humano e igualdad de género. Ahora se presenta una versión en formato de texto académico.
} 
del problema de desigualdad que existe entre las mujeres y hombres. La evidencia mostrada describe diferentes problemáticas que aquejan a las mujeres.

En el segundo apartado se traza el marco normativo imperante para la igualdad de género en México. Se parte de los tratados internacionales que el país ha reconocido, la creación de leyes de igualdad y los programas institucionales diseñados, así como el marco institucional que le da al municipio facultades en temáticas y políticas en la igualdad de género.

Finalmente, y con el enfoque de políticas públicas, se delinean elementos metodológicos para el diseño, aplicación y evaluación de las políticas de igualdad de género en el municipio. Resulta imprescindible mencionar desde este momento que los ayuntamientos desde sus capacidades institucionales y respectivas competencias, lo pueden hacer a través de los servicios públicos municipales, su facultad reglamentaria y algunos programas gubernamentales.

\section{La dimensión del problema: a manera de contextualización}

De acuerdo con la Organización de las Naciones Unidas 2012, las mujeres desempeñan dos tercios del trabajo mundial, obtienen sólo 10 por ciento de los ingresos y poseen menos del $1 \%$ de las propiedades en el mundo. Además de que se encuentran excluidas de la mayoría de los proyectos sociales y de la toma de decisiones de gobierno. En México esta situación no es la excepción, según el Instituto de Estadística, Geografía e Informática (INEGI, 2015), en 2013 el 69.8\% del trabajo remunerado lo desempeñan los hombres de 14 años y más, de la misma forma en todos los niveles de escolaridad las mujeres percibían menores ingresos que los hombres; por otro lado en 2010, en los hogares con jefatura masculina, el $91.3 \%$ el jefe era propietario de la vivienda, mientras que en los hogares con jefatura femenina sólo el $43 \%$ la jefa era la propietaria. Sobre el trabajo doméstico, en el año 2012, en el cuidado de personas el $73 \%$ lo desempeñan las mujeres, a su vez la responsabilidad del trabajo no remunerado (doméstico y de cuidados) recaía en la mujeres con el $62.2 \%$ del tiempo total de trabajo, en contraste, los hombres utilizaban el $76.2 \%$ del tiempo total de trabajo en trabajo de mercado. 
En cuanto a la representación política, y con datos de INEGI (2015), para 2014, la Cámara de Diputados estaba compuesta en un $62.4 \%$ por diputados hombres contra el $37.6 \%$ de diputadas mujeres; sin embargo la brecha se acentuó cuando se observan los porcentajes de quién preside el trabajo en las comisiones legislativas. El resultado fue que los hombres presidían el $76.8 \%$ contra el $23.2 \%$. En el caso de los municipios la brecha continuó: de cada 100 presidencias municipales sólo siete eran del sexo femenino, y dentro del cuerpo colegiado de regidores, de cada tres personas dos eran hombres en contraste con una mujer; de los síndicos de cada 10 sólo dos son del sexo femenino; y de los regidores en el país, con datos de la Encuesta Nacional de Gobierno, Seguridad Pública y Justicia Municipal 2009, (INEGI, 2010) existían 12579 hombres y únicamente 4757 eran mujeres regidoras.

La desigualdad se manifestó de nueva cuenta cuando, y de nuevo con base en datos de INEGI-2015, para el caso de la muerte de mujeres, la tasa de mortalidad por causa de cáncer de mama aumentó en los últimos años al pasar de 14.7 a 16.3 muertes por cada cien mil mujeres de 25 y más años de edad, entre 2001 y 2013; en contraposición con estos datos, en el país durante el año de 2013 el $55.9 \%$ de las defunciones correspondía a hombres. (Inmujeres, 2007). ${ }^{2}$

Precisamente en la vida cotidiana es donde se habla de oportunidades, y en ella resulta inevitable observar las brechas de género que existen; es decir, la desigualdad entre mujeres y hombres de las regiones y municipios del país, las cuales se origina por la discriminación. De persistir la exclusión en poco más de mitad de la población mexicana, los beneficios que generan el crecimiento y desarrollo económico para todos en igualdad de circunstancias seguirá siendo una cuenta pendiente. Por supuesto que lograr reducir la brecha entre las mujeres y hombres requiere de esfuerzos institucionales para movilizar voluntades, promover y redirigir políticas, normas, mecanismos, capacidades y recursos que contribuyan a la definición, aplicación, seguimiento y evaluación de las políticas y medidas necesarias.

\footnotetext{
${ }^{2}$ Este y otros factores explican porque hay más mujeres que hombres. Las causas determinadas conforme a este estudio son que los hombres mueren más que las mujeres por accidentes o agresiones, siendo el grupo de edad entre 15 a 29 años donde el total de muertos de hombres triplica al de mujeres, y el grupo de edad de 30 a 64 lo duplica. En suma se mueren más hombres que mujeres, jóvenes y en edad productiva, y por causas que no son enfermedades.
} 
Al respecto, los datos son contundentes y la encomienda se ve difícil ya que no sólo se necesita contar con políticas públicas y una normatividad con equidad de género, sino que dichas propuestas sean diseñadas, aplicadas y apropiadas por las mujeres y hombres y dentro del marco institucional para los municipios. A lo anterior se debe sumar que el entramado institucional mexicano es complejo a partir de que el sistema federal reconoce tres órdenes de gobierno el federal, estatal y municipal, así como al Distrito Federal y en el cual y en diversos temas de políticas públicas se reconocen en la Constitución Política de los Estados Unidos Mexicanos (CPEUM, 1999) la demandan de concurrencia, coordinación y cooperación intergubernamental. De tal modo que estar atento a las diversas competencias implicará tener en cuenta al sistema federal y al sistema de relaciones intergubernamentales condicionadas.

En el caso de la normatividad por disposición constitucional los municipios cuentan con competencias directas, y en específico con asuntos de servicios públicos señalados en la fracción III del artículo 115 de la Constitución mexicana, entre los cuales está el agua, alumbrado público, mercados, etc.; además de tener competencias concurrentes con los gobiernos federal y estatal, como es el caso en materia ecológica, donde existen competencias de los tres órdenes de gobierno, pero que se encuentran debidamente distribuidas en las leyes y reglamentos; y finalmente los municipios en algunas ocasiones coadyuvan con el gobierno federal o estatal en asuntos que por la urgencia y necesidad, aquellos no pueden atender suficientemente, como el caso de la educación y la salud.

Dichas competencias se encuentran definidas y atienden a diversas materias en la solución de la problemática. También cada orden de gobierno construye sus políticas públicas y define sus ordenamientos; para el caso de los gobiernos municipales ante la diversidad de los mismos (dos mil cuatrocientos cuarenta municipios en el país (INEGI, 2010), con el mismo número de visiones y maneras de diseñar e implementar sus herramientas jurídicas y de políticas públicas); resulta complejo definir políticas públicas generales que atiendan la problemática de género según sus competencias definidas en el artículo 115 Constitucional, si acaso se pueden construir algunas herramientas para el diseño y aplicación de las políticas públicas municipales, y la normatividad local con equidad de género para resolver las problemáticas que se presentan en este tema.

En los compromisos mundiales y en las distintas declaraciones 
nacionales e internacionales se destacaron valores fundamentales indispensables para el desarrollo humano sostenible: la igualdad, la solidaridad, la libertad, la responsabilidad común, la tolerancia y el respeto a la naturaleza. Véase el PNUD (Programa de las Naciones Unidas para el Desarrollo) como el paradigma del desarrollo humano tendiente a un desarrollo sostenible que tiene en Amartya Sen a su artífice teórico. También que en el año 2000, y en el encuentro celebrado en la sede de las Naciones Unidas, mediante la Declaración de Beijing el cual se le dio seguimiento a la IV Conferencia Mundial sobre la Mujer, se puso énfasis en el trabajo conjunto para lograr la equidad entre hombres y mujeres.

Derivado de lo anterior, el gobierno federal incluyó nociones como el desarrollo humano sustentable constituye el principio rector de los objetivos (PND, 2007) y después la perspectiva de género como una estrategia transversal para el desarrollo nacional, que servirá para garantizar la igualdad sustantiva entre mujeres y hombres (PND, 2013). ${ }^{3}$

Ante la complejidad del problema un punto de partida y supuesto general para decir que en México la igualdad de género existe, es si observamos que ésta se estipula en la Constitución Política de los Estados Unidos Mexicanos en su artículo 4: al señalar que el varón y la mujer son iguales ante la ley. No obstante, en este simple reconocimiento hay procesos internacionales previos o procesos nacionales simultáneos que denunciaron que la igualdad no se ha dado y el reto es hacerla realidad. De este modo, la perspectiva de género que se intenta aplicar de manera transversal a las políticas de equidad se ha definido como el tratamiento deliberado hacia situaciones de justicia dirigido a personas o grupos humanos que están sometidos a desigualdades por motivo de su sexo biológico (Paredes, 2006: 743). La situación anterior obliga a trazar el marco institucional que les permite a los ayuntamientos el impulso de políticas públicas con igualdad de género.

\footnotetext{
${ }^{3}$ Véase el Plan Nacional de Desarrollo 2007-2012, México, 2007, p. 18; y el Plan Nacional de Desarrollo 2013-2018, México, 2013.
} 


\section{La igualdad de género en el marco institucional}

El derecho a la igualdad entre hombres y mujeres está reconocido en diversos documentos internacionales, pero al final este derecho sólo se hará efectivo si hay políticas públicas y programas sociales que contribuyan a ello. No obstante, la travesía para que fueran reconocidos por los gobiernos tiene valor en sí debido al contexto en el que las mujeres, principalmente ellas, lucharon por el reconocimiento de tales derechos.

La reforma de gran envergadura en materia de derechos humanos fue la que reconoció la no discriminación de persona alguna motivada por género o preferencias sexuales, como un factor determinante en el goce de los derechos humanos. Esta reforma se publicó en el Diario Oficial de la Federación (DOF) con fecha 10 de junio de 2011 y trajo como consecuencia la obligatoriedad del Estado mexicano para que buscara su cabal cumplimiento y fuera acorde con los compromisos en la materia que previamente se habían firmado en diversos tratados internacionales. La reforma obligó a que los tres órdenes de gobierno a su observancia así como que implementaran políticas públicas nacionales, estatales y municipales.

\section{a) Los tratados internacionales}

El itinerario para que los gobiernos nacionales reconocieron y firmaron como derecho la igualdad entre hombres y mujeres, inició en diciembre de 1948 con la Declaración Universal de los Derechos Humanos (DUDH, 1948). Dicha declaración es la base internacional primigenia de la perspectiva de género en las agendas públicas y la acción gubernamental en México. En la declaración se reconocieron los derechos fundamentales entre los hombres, los cuales se basan en la dignidad y el valor de la persona humana, y quedó asentada la igualdad de derechos de hombres y mujeres para promover el progreso social y elevar el nivel de vida dentro de un concepto más amplio de la libertad (DUDH, 1948).

Poco tiempo después, en marzo de 1953, la Convención sobre los Derechos Políticos de la Mujer (CDPM, 1953) reconoció que toda persona tenía derechos a participar en el gobierno de su país. En la generalidad de este derecho no sólo se denunció la exclusión de las mujeres en la vida política y pública de muchos países sino que se señaló la desigualdad por razón del sexo biológico. La convención 
si bien reconocía que las mujeres podrían participar directamente o por conducto de sus representantes libremente escogidos en la vida política, abogó por que hubiera oportunidades de ingreso en el servicio público de los países que lo firmaban (CDPM, 1953).

Una década después, en diciembre 1966, diversos gobiernos nacionales firmaron el Pacto Internacional de Derechos Civiles y Políticos y su protocolo facultativo (PIDCP, 1966). Con este instrumento y conforme a los principios enunciados en la Carta de las Naciones Unidas (libertad, justicia y paz), se reconoció la dignidad inherente a todos los miembros de la familia humana y de sus derechos iguales e inalienables. De igual modo, se estableció que estos derechos se derivan de la dignidad inherente a la persona humana y que no podría realizarse el ideal del ser humano libre sin que se abatiera el temor y la miseria a menos que se crearan condiciones que permitieran a cada persona gozar de sus derechos civiles y políticos, tanto como de sus derechos económicos, sociales y culturales (PIDCP, 1966).

La travesía en la demanda de la igualdad de la mujer con respecto a los hombres se hizo más fuerte cuando en diciembre de 1979 se llevó a cabo la Convención sobre la Eliminación de todas las formas de Discriminación de la Mujer (CEDAW por sus siglas en inglés, 1979). Poco tiempo después, en septiembre de 1981, entró en vigor su protocolo facultativo. Con ambos instrumentos se intentó, al menos jurídicamente, que los compromisos adquiridos por los gobiernos fueran vinculantes; además de contener un abanico de derechos más amplio, que a la vez fueran específicos con respecto a los documentos internacionales previamente creados. La convención denunció la discriminación y privación de derechos hacia las mujeres por lo que se establecieron principios y medidas universales para que gozaran de igualdad de derechos y garantías (CEDAW por sus siglas en inglés, 1979).

En esta dirección el hemisferio americano hizo lo propio, cuando en abril de 1948 se llevó a cabo la Convención Interamericana titulada "Concesión sobre los derechos civiles a la mujer" (CDCM, 1948). La convención entró en vigor en julio de 1954, y en ella la mayoría de las repúblicas americanas, inspiradas en los principios de la justicia, concedieron derechos civiles a la mujer. Poco tiempo después, y posteriormente al reconocimiento de los derechos civiles, los países americanos reconocieron que la mujer tenía derecho a un tratamiento político igual que el del hombre en la Convención Interamericana "Concesión sobre los derechos políticos de la mujer" (CDCM, 1948). 
Las cuatro conferencias mundiales de la mujer fueron los foros internacionales y los documentos que de ellas se desprendieron donde se trataron los temas relacionados con las desigualdades que sufrían las mujeres. La primera se llevó a cabo en México en 1975; la segunda en Copenhague en 1980; la tercera en Nairobi en 1985; y la cuarta en Beijing en 1995. La más relevante fue la de Beijing pues en ella se buscó la incorporación de las prescripciones de las convenciones internacionales en las legislaciones nacionales y estatales, así como velar por su debido cumplimiento. También en esta convención surgió la necesidad de incorporar la perspectiva de género en las políticas, programas, presupuestos y gestión de instituciones públicas. ${ }^{4}$

\section{b) Las políticas federales}

Ante tal panorama es necesario recordar que si bien la Constitución Política de los Estados Unidos Mexicanos reconoce en su artículo 4 la igualdad entre los hombres y las mujeres, así como que el gobierno federal ha suscrito los derechos y garantías reconocidas en tratados y convenciones internacionales, éstos requieren de leyes secundarias, instituciones e instrumentos programáticos para hacerlos efectivos (CPEUM, 1999).

En este sentido, el 2 de agosto de 2006 se creó la Ley General para la Igualdad entre Mujeres y Hombres, publicada el 2 de agosto de 2006 en el Diario Oficial de la Federación (LGIMH, 2006) cuyo objetivo fue garantizar la igualdad entre mujeres y hombres, y proponer los lineamientos y mecanismos institucionales que orienten a la nación hacia el cumplimiento de la igualdad en los ámbitos público y privado promoviendo el empoderamiento de las mujeres (LGIMH, 2006). ${ }^{5}$

Otra ley importante en el reconocimiento de la problemática de las mujeres es la Ley General de Acceso de las Mujeres a una Vida Libre de Violencia, publicada el $1^{\circ}$ de febrero de 2007 en el DOF, que tiene como objetivo la coordinación entre los tres órdenes de gobierno para prevenir, sancionar y erradicar la violencia contra las mujeres. En este sentido, el artículo 49 de la ley señaló que las entidades federativas

\footnotetext{
${ }^{4}$ De entonces a la fecha ha habido otros documentos o tratados internacionales que si bien retoman lo de la convención de Beijing son más específicos o son de carácter regional. Un ejemplo del primero es "Metas de milenio" de septiembre del año 2000 donde se establecieron 8 metas, entre las que destacó la que se refería a promover la igualdad entre los géneros y la autonomía de la mujer. Las metas estaban propuestas para alcanzarse en el 2015. ${ }^{5}$ El término empoderamiento se refiere a la necesidad de que las mujeres ganen poder, según el Glosario de Género (Inmujeres, 2007, p. 58).
} 
deberán implementar políticas públicas en la materia y ejercer su facultad reglamentaria para la aplicación de esta ley. Para el caso de los municipios el artículo 50 de la ley establece que deberán crear e implementar la política pública acorde a los programas nacionales y estatales en la materia, orientada a erradicar la violencia en contra de las mujeres.

En este contexto de ordenamientos jurídicos en la materia, la Ley del Instituto Nacional de las Mujeres se creó el 12 de enero de 2001, y tuvo como objetivo promover y fomentar las condiciones que posibilitaran la no-discriminación, la igualdad de oportunidades y de trato entre los géneros; el ejercicio pleno de todos los derechos de las mujeres y su participación equitativa en la vida política, cultural, económica y social del país con criterios de transversalidad de género en políticas públicas, programas y acciones gubernamentales. La ley al ser programática, es decir que se encarga de la implementación y ejecución de programas de gobierno en la materia y el Instituto Nacional de las Mujeres al inscribirse como una dependencia y organismo dentro del enfoque de las políticas públicas, tiene como finalidad fortalecer a las dependencias de la administración pública federal para incorporar la equidad de género en ellas y fortalecer los vínculos entre los tres poderes federales: Ejecutivo, Legislativo y Judicial.

Pero quizá la acción más importante fue la creación del Programa Nacional para la Igualdad entre Mujeres y Hombres (Proigualdad, 2009), publicado en el Diario Oficial de la Federación de fecha 18 de agosto de 2009, que le da operatividad y aplicabilidad a los ordenamientos jurídicos, toda vez que al contar con acciones específicas actualiza los preceptos señalados en la normatividad. El programa buscó garantizar los derechos humanos de las mujeres, la no discriminación, el acceso a la justicia y a la seguridad, así como fortalecer las capacidades de las mujeres para su fortalecimiento económico a favor de generar mayores oportunidades para su bienestar y desarrollo; promover la igualdad de oportunidades; eliminar cualquier discriminación por motivos de género y que mujeres y hombres ejerzan sus derechos. ${ }^{6}$

\footnotetext{
${ }^{6}$ Lo anterior a través de siete objetivos estratégicos: Objetivo estratégico 1: Institucionalizar una política transversal con perspectiva de género en la Administración Pública Federal, y construir los mecanismos para contribuir a su adopción en los poderes de la unión, en los órdenes de gobierno y en el sector privado. Objetivo estratégico 2: Garantizar la igualdad jurídica, los derechos humanos de las mujeres y la no discriminación, en el marco del estado de derecho. Objetivo estratégico 3: Garantizar el acceso de las mujeres a la justicia, la seguridad y la protección civil. Objetivo estratégico 4: Garantizar el acceso
} 
Por lo tanto por equidad de género se debe entender el principio de justicia e igualdad sustantiva que reconoce las diferencias sociales. También la perspectiva de género es una herramienta metodológica que ayuda a detectar y analizar el impacto diferenciado entre mujeres y hombres de las políticas y acciones gubernamentales, o de las organizaciones no gubernamentales.

Aunque cabe decir que en la perspectiva de género no se deben perder de vista las diferencias que existen entre los distintos grupos de mujeres: edad, estado civil, etnia, raza, clase social, así como las diferencias geográficas y regionales, entre otros. Lo que hace la perspectiva de género es ayudar a identificar y analizar las condiciones y posiciones sociales de mujeres y hombres. Si se hace una buena lectura y se aprovechan las capacidades institucionales y/o de innovación que permite el Artículo 115 constitucional a los municipios mexicanos, pueden existir acciones y experiencias que podrían ser el inicio y la base para impulsar un cambio. De tal modo que el marco constitucional en combinación con la perspectiva de género y el enfoque de políticas públicas ofrecerían herramientas para impulsar la igualdad de género. Pero antes se debe delinear el marco jurídico del municipio mexicano que proporciona algunas luces para la innovación.

\section{c) El marco constitucional del municipio}

En cuanto al marco constitucional, el artículo 115 constitucional señala que cada municipio en México será gobernado por un ayuntamiento de elección popular directa (Fracción I). Con este mandato no sólo se reconoce al municipio como un orden de gobierno sino que la función de gobernar implica contar con el mandato de promover, respetar, proteger y garantizar los derechos reconocidos a las personas y por lo tanto, ser una instancia competente para impulsar el desarrollo local a través del sistema de planeación'(CPEUM, 1999).

\footnotetext{
de las mujeres a una vida libre de violencia. Objetivo estratégico 5: Fortalecer las capacidades de las mujeres para ampliar sus oportunidades y reducir la desigualdad de género. Objetivo estratégico 6: Potenciar la agencia económica de las mujeres en favor de mayores oportunidades para su bienestar y desarrollo. Y; Objetivo estratégico 7: Impulsar el empoderamiento de las mujeres, su participación y representación en espacios de toma de decisión en el Estado y consolidar la cultura democrática (Proigualdad, 2009).

${ }^{7}$ Con esta plataforma las políticas públicas y las acciones de gobierno encuentran instrumentos específicos que además de incluirse en la planeación estratégica y el desarrollo local, deben tener la perspectiva de género. Un ejemplo es el Plan Municipal de Desarrollo; la creación de programas especiales de Igualdad entre mujeres y hombres, programas especiales de cultura
} 
La Carta Magna mexicana es enfática cuando dice (artículo 115) que los ayuntamientos tienen facultades para aprobar los bandos de policía y gobierno, los reglamentos, circulares y disposiciones administrativas de observancia general dentro de sus respectivas jurisdicciones (Fracción II). De esta disposición constitucional se desprende que el municipio, y en tanto orden de gobierno, tiene facultades exclusivas para la creación de Reglamentos Municipales y demás ordenamientos jurídicos que normen al gobierno, sus funciones y servicios, así como las relaciones entre sus habitantes y para con el gobierno mismo. Además de que es competente para aprobar un reglamento del gobierno municipal que norme sus relaciones internas, la función pública y los servicios, sus relaciones para con los habitantes del municipio mujeres y hombres en igualdad de derechos, así como para las personas en tránsito por la municipalidad (CPEUM, 1999).

De tal modo que cada municipio del país cuenta con un "marco jurídico municipal" que debería estar impregnado de la perspectiva de género y orientarse con el enfoque de políticas públicas y el desarrollo local. Desde su capacidad reglamentaria los municipios son competentes para organizar su administración, de acuerdo a sus propias especificidades y presupuesto. De igual forma, los ayuntamientos están facultados para crear reglamentos sobre los derechos y obligaciones de las mujeres y los hombres que laboran en la administración pública, así como regular las condiciones generales de trabajo de las servidoras y servidores públicos (CPEUM, 1999).

Pero con mayor claridad, están facultados constitucionalmente para aprobar reglamentos que organicen la administración pública municipal para poder crear Instancias o dependencias que tengan como objetivo atender y empoderar la mujer en la municipalidad. La figura jurídico-administrativa podrá variar en el sentido de si es centralizada, desconcentrada o descentralizada. ${ }^{8}$

institucional y los Programas Operativos Anuales (Poas) o Programas Anuales de Resultados (PAR) encaminados a resolver problemáticas específicas como la violencia contra las mujeres, por citar un ejemplo; la creación de estancias infantiles para las madres que trabajan o jefas de familia; orientación jurídica; promoción y desarrollo de habilidades, entre otras. ${ }^{8}$ Esto dependerá de las características de cada municipio: de sus recursos disponibles, su estructura administrativa y sobre todo de las problemáticas identificadas de género; a saber cada una de las figuras tiene atributos se puede decir que: a) la centralizada se encuentra sujeta a las decisiones del ayuntamiento, no tiene capacidad de obligarse y contratarse, de organizarse por sí sola, de crear sus propios programas y de gestionar sus propios recursos; b) la desconcentrada no puede hacer todo lo anterior con excepción de crear sus propios programas; y c) la descentralizada puede ejercer todas las atribuciones anteriores, y solo responder a una política general y subsidio del ayuntamiento. 
También los gobiernos municipales son competentes para aprobar reglamentos municipales que regulen la prestación de los servicios públicos, esto es, las atribuciones de la autoridad para el suministro y los derechos y obligaciones de los habitantes para con los servicios públicos. En este contexto, se podría reglamentar el acceso a los servicios y al control de recursos que combatan la desigualdad que sufren las mujeres en la sociedad que es dónde más pueden incidir en las políticas públicas: servicios públicos, reglamentación, planes y programas gubernamentales de orden y origen municipal. Las páginas esbozarán lo que el marco normativo permite y hace posible como políticas públicas municipales con perspectiva de género.

\section{Políticas públicas locales con perspectiva de género}

A partir de la década de 1980, en México se instauró en el lenguaje político y académico el término de políticas públicas. Tales locuciones se insertaron en un contexto de democratización política, y el término de políticas públicas contribuyó a renovar el discurso político y gubernamental (Aguilar, 2005), al mismo tiempo se convirtió en un marco para el análisis de la función pública (Parsons, 2007). Pero el enfoque de políticas públicas supone la existencia de un sistema político democrático en el cual las soluciones difícilmente pueden ser impuestas de forma unilateral.

De ahí que la política pública y la forma que adopta la acción gubernamental: sus decisiones y programas, sean un factor en el que se deba observar cómo los gobiernos procesan las múltiples demandas e interactúan con una constelación de instituciones y actores/as políticos, sociales y económicos. Desde tales consideraciones, el asunto de la equidad de género, con sus elementos constitutivos y normativos, se vuelve fundamental en las organizaciones públicas porque el cometido es impulsar y promover la igualdad entre hombres y mujeres.

Surge así una veta que es discursiva y analítica: las políticas públicas con perspectiva de género. De ahí que el sentido imperativo es que el Estado, y su acción gubernamental y los diversos programas con los que se manifiesta hacia la sociedad, sean incrustados transversalmente de la noción que busca promover y garantizar la igualdad de género. 
Pero en el complejo entramado institucional estatal qué papel o función podrían tener los gobiernos municipales. Un punto de partida sería decir que lo harían participando y formulando políticas con equidad de género. Empero, antes de ello no se deben perder de vista el marco institucional y normativo con el que cuentan. Ya se vio que los gobiernos municipales tienen un marco internacional que ofrece, además de ser ley, elementos conceptuales donde se asienta el derecho a la igualdad de las mujeres con respecto a los hombres. También cuentan con un marco programático que intenta hacer factibles y prácticos estos derechos, y desde luego un marco constitucional que más adelante será descrito.

Si bien es cierto que las políticas de género exitosas son aquellas que cuentan con la participación de las mujeres y hombres del municipio, ${ }^{9}$ y que son el medio por el cual el Estado puede eliminar la desventaja para las mujeres, esto no será posible si los institutos o instancias de las mujeres en México (federal, estatales y municipales) no identifican y precisan los problemas de inequidad de las mujeres en la administración pública y el entorno local donde se asienta el municipio respectivo. En el análisis de la problemática de las mujeres en un ámbito local y al comenzar se debe iniciar con el diseño de las políticas públicas debe iniciar con un diagnóstico, cuya finalidad es estrictamente instrumental y descriptiva. Su utilidad principal es impulsar el enfoque de género en el diseño e implementación de las políticas públicas municipales. Un diagnóstico es un ejercicio de investigación y tiene que cumplir con tres elementos metodológicos esenciales: un enfoque analítico, un conjunto de técnicas de investigación y un conjunto de fuentes de información. ${ }^{10}$

El uso combinado de técnicas cualitativas y cuantitativas permitirá obtener resultados integrales, y por lo tanto un potencial descriptivo para diagnosticar problemas públicos. ${ }^{11}$

9 Se ha encontrado en estudios de caso que en la formulación de políticas públicas de género la denominada "gestión municipal participativa", es la mejor manera de aplicarlas para que sean efectivas (véase Bassols y Massolo, 2003, pp. 16 y 17). ${ }^{10}$ Dada la complejidad cultural que los problemas de desigualdad como consecuencia del género pudieran tener, el enfoque de investigación para el desarrollo de un diagnóstico tendría que ser mixto, es decir que utilicé equilibradamente tanto aspectos de investigación cuantitativa como cualitativa. La razón principal es que las problemáticas de género desde cualquier dimensión temática (económica, social y política) difícilmente serían observables a partir de un enfoque de investigación simple. ${ }^{11}$ A pesar de la facilidad con la que se pueden obtener datos estadísticos censales a nivel municipal en México, es recomendable que se utilicen equilibradamente técnicas de recolección de datos que capten información de fuentes indirectas, sin dejar de lado la recolección de información 
Dada a la naturaleza de los diagnósticos, su alcance es exploratorio en cuanto la delimitación de la situación de las mujeres en el municipio determinado, y descriptivo en cuanto a la delimitación del contexto en el que se inscribe. Un entorno analítico de las características anteriores, hará más fácil y/o pertinente la incorporación del enfoque de género en la gestión municipal. ${ }^{12}$

El fin es conformar políticas públicas locales con una perspectiva de género y adecuar las normas y programas para que sean redistributivos y focalizados. Para desarrollar este tipo de políticas se requieren múltiples acciones que incluyen en el largo plazo cambios estructurales en las organizaciones, la incorporación y transversalización del enfoque de género en todas las acciones de gobierno, y por último la institucionalización de la perspectiva de género en la administración pública. En el corto plazo se hace necesario transformar la forma como se conciben los problemas, rediseñar la estructura de la administración pública, realizar cambios institucionales. Adicionalmente, diseñar políticas de acciones compensatorias para impulsar el desarrollo de las mujeres, además de herramientas que permitan a los generadores de las políticas lograr esta encomienda.

En suma, en el municipio mexicano se pueden diseñar e implementar diferentes instrumentos normativos y políticas públicas que se conviertan en facilitadores de la equidad de género y/o de los procesos como: a) la prevención de la delincuencia y la seguridad ciudadana; b) contra la violencia de género; c) de salud sexual y reproductiva; d) la generación de ingresos; y e) en temas de agua y el saneamiento. Esto es necesario y urgente porque sociológicamente la discriminación y desigualdad de la mujer es estructural. De tal modo que los gobiernos municipales por medio de sus atribuciones normativas y reglamentarias pueden cimentar bases de acción afirmativa que contribuyan a combatir la desigualdad. La acción de los gobiernos municipales es enriquecer el marco normativo al estar más atentos a las demandas sociales, las necesidades y obstáculos que

de fuentes directas. Las fuentes de información indirecta pueden aportar elementos de carácter cuantitativo o cualitativo que se tendrían que balancear con la aplicación de técnicas de investigación directa igualmente equilibradas: como son las encuestas y las técnicas cualitativas. 12 Se debe entender por gestión pública municipal “...la formulación de planes y políticas e implementación de programas, proyectos y obras que incluyan las demandas comunes y específicas de mujeres y hombres en su diversidad. De esa manera se busca revertir situaciones de inequidad e injusticia, tomando en cuenta la condición de género, las socioeconómicas y culturales de las personas, así como sus necesidades respecto al territorio y los servicios, tanto para desarrollar sus actividades como sus aspiraciones personales" (véase Derechos Humanos de las Mujeres, 2004). 
dificultan la participación activa de las mujeres en todos los ámbitos del quehacer humano. Luego entonces el papel institucional del municipio es coadyuvar al pleno ejercicio de la igualdad de derechos, oportunidades, recursos y libertades.

\section{a) Los servicios públicos municipales}

Como se notó páginas atrás, la Constitución federal da luces de la forma en cómo institucionalmente el ayuntamiento puede impulsar la igualdad de género con programas y acciones concretas, toda vez que las competencias del municipio resultan muy concretas acerca de la posible atención con las mujeres y hombres de su territorio; es decir, este ámbito se convierte en el gobierno de los vecinos/as cuando atiende cosas tan puntuales como son: los servicios públicos de agua potable, alumbrado, calles, parques, y en la función pública de ordenar el territorio y la ecología de su territorio.

Por ejemplo, la Fracción II, Inciso a del Artículo 115 establece las bases generales de la administración pública municipal y del procedimiento administrativo. En él se mencionan los medios de impugnación y los órganos para dirimir las controversias entre la administración y los/as particulares, con sujeción a los principios de igualdad, publicidad, audiencia y legalidad. De esta disposición se podría decir que los gobiernos municipales están facultados para crear instancias municipales que se orienten a la promoción de la justicia de género a través de la creación de Juzgados Municipales como es el caso del Estado de Jalisco. El papel o la función de estas instancias sería la de conocer, calificar e imponer sanciones administrativas municipales que procedan por faltas o infracciones a los ordenamientos municipales; conciliar a los vecinos/as en los conflictos que no sean constitutivos de delito, ni de la competencia de los órganos judiciales o de otras autoridades; brindar atención jurídica gratuita y promover programas para la difusión de los conocimientos básicos sobre derechos de las mujeres y sus procedimientos legales de mayor interés para las mujeres; y crear programas de mediación familiar.

Con mayor precisión los ayuntamientos podrían, por ejemplo, en temas de agua potable, alcantarillado y disposición de sus aguas residuales, implementar acciones en las que la prestación de los servicios públicos esté destinada a la satisfacción de las necesidades básicas y mejorar la calidad de vida de la población. La prestación del servicio y su reglamentación podría originar programas que se dirijan 
a familias y las comunidades donde la condición de la mujer implique situaciones de desigualdad y marginalidad..$^{13}$

En cuanto al alumbrado público, si bien es una actividad técnica, el servicio está destinado a satisfacer una necesidad en la que la iluminación podría desfavorecer la comisión de delitos, violencia o agresiones contra las mujeres.

La aseveración de que el servicio de recolección de residuos es una actividad técnica y medioambiental, su importancia, y desde una perspectiva de género, radica en que el aseo de calles y lugares públicos implica una cuestión de salud para los integrantes del hogar y para las madres jefas de familia. La eficiencia y eficacia de la autoridad municipal en la prestación de este servicio impacta directamente en la calidad de vida de las mujeres y hombres.

En la cartera de servicios públicos que son facultad o atribución de los municipios también está la de los mercados y centrales de abasto. Este servicio que podría ser concebido desde el punto de vista técnico como aquel destinado a satisfacer el aprovisionamiento de productos y servicios indispensables para la alimentación y la economía doméstica, podría generar programas y apoyos a mujeres.

En el servicio de panteones los ayuntamientos pueden diseñar mecanismos jurídico-administrativos a efecto de facilitar los trámites mortuorios a las cónyuges supervivientes, muy en especial cuando las madres de familia quedan al frente de los hogares. El acceso al consumo de productos básicos debe ser una de las preocupaciones fundamentales del municipio. Sobre todo, generar condiciones que faciliten el acceso de las mujeres dueñas de casa (trabajo doméstico) y la dinámica del cuidado de hijos/as y dependientes. La implementación de políticas públicas municipales para el acceso a la adquisición de vivienda para las mujeres en situación de vulnerabilidad y considerando que muchas de ellas no pueden tener acceso a esto debido a que no cuentan con trabajos formales con prestaciones. Así como facilitar el acceso con horarios, cercanía y seguridad adecuada para mujeres que trabajan.

De mantenimiento de calles, parques y jardines y su equipamiento, e insertados en la noción de rescate y uso de espacios públicos, son determinantes para que las mujeres puedan acceder a estos servicios

13 El suministro del agua potable en la municipalidad es un componente básico de la vida y elemento determinante para conocer la situación de las mujeres dentro de la comunidad. 
públicos con la garantía de que serán respetadas en sus derechos y con ello en su integridad física y moral.

Finalmente, el servicio de seguridad pública el cual es una función a cargo de los órdenes de gobierno, es uno de los pilares para garantizar el ejercicio de los derechos de las personas. En América Latina, uno de los desafíos más importantes de la seguridad es la violencia de género, expresada no sólo en el ámbito de lo privado y lo doméstico, sino también en la vida pública y en la ciudad, atentando directamente contra la integridad de las mujeres y la igualdad de oportunidades (ONU-HABITAT, 2009: 26).

De ahí que para enfrentar la violencia de género y la violencia familiar, la acción del gobierno municipal sea indispensable, pues a través de planes municipales, acciones y reformas a los reglamentos municipales se daría un paso para erradicar la violencia contra las mujeres, las niñas, los niños, los discapacitados/as y adultos/as mayores. En el ámbito local se podrían hacer acciones que aborden los temas como la violencia contra la mujer, centros de atención, programas de reeducación de agresores, refugios para víctimas, y coordinación con organizaciones de la sociedad civil (ONU-HABITAT, 2009: 26).

\section{b) Otros mecanismos institucionales y gestión pública}

A la cartera de servicios municipales que por disposición constitucional los ayuntamientos deben proporcionar a la población, hay otros mecanismos institucionales en los que se puede aprovechar la perspectiva de género y el enfoque de políticas públicas para impulsar la igualdad entre las personas.

Uno de ellos es el que se desprende, previo acuerdo entre los ayuntamientos, de la capacidad de coordinarse y asociarse los municipios para la eficaz prestación de los servicios públicos o el mejor ejercicio de las funciones que les correspondan. En este caso y tratándose de la asociación de municipios de dos o más Estados, deberán contar con la aprobación de las legislaturas de los Estados respectivos (véase el artículo 115, Fracción III, Inciso c de la constitución federal). Esta posibilidad se desprende ante el fenómeno de que un ayuntamiento por sí sólo no puede atender las demandas en el suministro de los servicios por las limitaciones presupuestales de los gobiernos municipales. Con esta figura jurídica se fortalece la capacidad institucional para que los gobiernos municipales se asocien mediante estrategias de cooperación y coordinación a efecto de 
responder de forma más efectiva a las demandas sociales.

En este contexto y, desde la perspectiva de género, el presupuesto podría ser producto de una análisis cuyo diagnóstico parta de un enfoque de género, además de soportar los objetivos plasmados en el Plan Municipal de Desarrollo con Igualdad de Género que fije el monto de recursos destinado al empoderamiento de las mujeres, etiquetando recursos dedicados al avance de las mujeres en todos los ámbitos de la gestión pública.

Finalmente, los municipios, en los términos de las leyes federales y estatales, están facultados para formular, aprobar y administrar la zonificación y planes de desarrollo urbano municipal (Artículo 115, fracción $\mathrm{V}$ de la Constitución federal). En este rubro la gestión del territorio es competencia constitucional del gobierno municipal al contar con las atribuciones para aprobar los reglamentos municipales, crear instancias administrativas e implementar políticas para planear, controlar y vigilar el uso del suelo y, con ello, impulsar el desarrollo sostenible de su municipalidad (CPEUM, 1917).

Cabe decir que la falta de reglas formales para el ordenamiento del territorio impacta directamente en la calidad de vida de las mujeres y de los hombres de una localidad y de sus generaciones por venir. De tal modo que determinar el lugar para la creación de nuevas colonias, la infraestructura para el suministro de los servicios como el agua, el alumbrado público, pasando por el otorgamiento de licencias para construir, hasta el cuidado y protección de áreas verdes y zonas de reserva, resulta de vital importancia para la sana convivencia de mujeres y hombres; en sí misma la planeación y gestión urbana es muy compleja por la dinámica económica que genera la demanda de vivienda y, los intereses políticos y de control sobre el uso del suelo, de tal forma que la inexistencia de reglas formales como los planes de ordenamiento territorial y sus respectivos planes parciales de zona,${ }^{14}$ contribuye a la discrecionalidad del municipio en el manejo del territorio y deja al ciudadano(a) en un estado de indefensión.

\section{c) La perspectiva de género en los reglamentos municipales}

Como se puede apreciar, los gobiernos municipales podrían incidir en la igualdad de género. Esto es así porque cuenta con las

\footnotetext{
${ }^{14}$ Para el caso de Jalisco, el Código Urbano establece como instrumentos normativos municipales el denominado Programa Municipal de Desarrollo Urbano, planes de desarrollo urbano de centro de población y planes parciales de desarrollo urbano.
} 
herramientas conceptuales y normativas para ello. Sin embargo, la perspectiva de género está ausente en la práctica gubernamental y política porque hay resistencia sociocultural al cambio institucional y/o no se cuenta con la experiencia o ejemplos concretos que ilustren cómo se puede impulsar la igualdad de género desde el municipio. Para lograr dicho cambio institucional es necesario primero que los y las ediles estén convencidos de ello, sin embargo por el desconocimiento del tema, la falta de sensibilidad, la visión a corto plazo, hace que no se implementen los instrumentos, aunado a que a la burocracia tampoco le interesa; es decir, no basta con crear instrumentos que impulsen la equidad de género, sino que deben de existir recursos económicos y materiales para hacerlo, y personal que cuente con el conocimiento en la materia.

Las fases que contempla el proceso reglamentario municipal: Iniciativa, dictamen, discusión, votación y aprobación, publicación, vigencia y difusión; y concretamente el profundo diagnóstico que implica ayudaría a comprender la importancia del gobierno municipal en el impulso de la igualdad de género. Para ello se debe partir de la premisa de que los cambios o creación de ordenamientos municipales, y en tanto cuerpos normativos, pueden accionar en beneficio de la igualdad entre mujeres y hombres. Al menos el hecho de que constitucionalmente el artículo 115 contemple como atribución municipal la competencia reglamentaria es porque puede incidir en el entorno sociocultural y por lo tanto puede incidir en el cambio institucional.

Dentro del proceso reglamentario del municipio cabe recalcar la importancia de sus principales fases: en la primera fase se tiene que hacer un diagnóstico; la justificación de la igualdad de género en la normatividad municipal debe comenzar a través de la elaboración de un profundo análisis en el que se haga visible la desigualdad de las mujeres con relación a los hombres dentro de la organización y el entorno municipal. La información debe segmentarse y aglutinarse en temas, áreas y/o dependencias que den cuenta de las necesidades y problemáticas de las mujeres y hombres del municipio. La planeación democrática y la participación de mujeres y hombres del municipio son necesarias para este fin.

La segunda fase es de diseño. En esta fase la creación de los temas de interés deben ser normados en los ordenamientos municipales. Las funcionarias/os municipales y asesoras(es) de las(os) ediles deben de estar capacitadas(os) en el tema de género y contar con 
los espacios y herramientas teórico-metodológicas que soporten el diseño de propuestas.

La tercera fase es de implementación. En ella se debe atender la normatividad para procurar la igualdad entre mujeres y hombres, de las funcionarias/os municipales de todas las dependencias desde mandos directivos hasta los operativos. Además de estar formados en el tema de género y conocer los alcances de aplicar correctamente estos preceptos. De la misma forma cada dependencia municipal debe contar con los recursos humanos, materiales y económicos para cumplir con los mandatos normativos para la igualdad de género, sino de nada serviría contar con ellos. Se tiene así el inicio de una cultura institucional incluyente que haría viable su aplicación.

En la capacidad reglamentaria del ayuntamiento para incluir la perspectiva de género se debe atender los siguiente puntos: 1. Un lenguaje incluyente; 2 . La creación y atención de las instancias municipales de las Mujeres para que emitan su posición o pronunciamiento en lo que hagan otras dependencias municipales; 3. Contengan acciones positivas de igualdad entre hombres y mujeres; 4 . Se obligue e incorpore en los ordenamientos normativos la equidad de género; 5 . Se prevean las necesidades presupuestales para planes y programas de igualdad; 6 . Se incentive la igualdad de hombres y mujeres en empleos del gobierno municipal; 7 . Se estipulen porcentajes mínimos de incorporación de mujeres dentro del gobierno municipal; y 8 . Se elimine en la reglamentación un lenguaje sexista y estereotipado.

\section{A manera de cierre}

Con el trazo anterior se puede decir que el ayuntamiento cuenta con una base para impulsar la igualdad de género. Cierto habrá que tener en cuenta las diferentes capacidades institucionales, financieras y los contextos locales de los municipios, pero se pueden diseñar e implementar programas con enfoque de género y/o crear organismos. Hay tratados internacionales, un marco normativo constitucional y federal que da cuenta de ello. El reto es hacer la armonización normativa que hay en el Estado mexicano realidad y para ello el 115 Constitucional tiene una parte instrumental. De esta correlación se deben pensar la acción gubernamental desde un enfoque de 
políticas públicas como un elemento innovador para el análisis de las problemáticas de desigualdad y construcción de respuestas viables que permiten abatir las desigualdades.

De tal modo que el municipio se puede volver un ámbito de gobierno relevante en el caso de los servicios públicos, pero también en la coordinación intergubernamental que fortalezcan la hacienda municipal, impulsen la participación ciudadana y logren un mejor desarrollo urbano y medio ambiente, entre otros. En este sentido el artículo 115 constitucional y las leyes de igualdad y contra la violencia, otorgan a los municipios espacios de coordinación que puede y en todo caso debe intervenir como ya se estableció, todo conforme sus competencias. El marco institucional puede parecer insuficiente pero impacta directamente en la vida de las personas por ejemplo con la participación ciudadana y la prestación de los servicios públicos, que otorgan a las personas el derecho a participar en las acciones del municipio.

En el presente lo que hay son retos y oportunidades, y ante todo se debe lograr un cambio institucional que implica la transformación de creencias y valores. Lo anterior es una empresa que se logra en el mediano y largo plazo, pero en el corto y debido a los problemas inmediatos del gobierno municipal y el tiempo reducido para la gestión pública, actores que ocupan sus estructuras de autoridad deben impulsar acciones concretas y específicas. Finalmente, la oportunidad más importante es que los propios hombres y mujeres de los municipios asuman su responsabilidad ante la desigualdad que existe, la problemática que cada vez se presenta más, que solo se logra con educación e incentivos positivos o negativos que la misma sociedad en su conjunto implemente.

Aguilar Villanueva, Luis F. (2005). "Las políticas públicas: su aporte", Adrián Acosta (coord.) Democracia, desarrollo y políticas públicas, Guadalajara, CUCEA-Universidad de Guadalajara, pp. 19-31.

Paredes, Rosa (2006). "Políticas públicas, pobreza y equidad de género". Espacio Abierto, 15 (4): 741-757.

Parsons, Wayne (2007). Políticas públicas. Una 
introducción a la teoría y la práctica del análisis de políticas públicas, México, FLACSO-Mino y Dávila editores.

\section{Documentos y leyes}

CUEJ (2008). Código Urbano para el Estado de Jalisco. Guadalajara. Periódico Oficial "El Estado de Jalisco", Guadalajara, Jalisco, México, 27 de septiembre.

CPEUM (1999). Constitución Política de los Estados Unidos Mexicanos. Diario Oficial de la Federación, México D.F., el documento consultado es del 23 de diciembre que contempla la reforma municipal.

DHM (2004). Derechos Humanos de las Mujeres. Diagnóstico sobre la situación de los derechos humanos en México. Oficina del Alto Comisionado de las Naciones Unidas para los Derechos Humanos en México, México, Mundi-Prensa.

DSLIG (2009).Desarrollo Local con Igualdad de Género. Volumen 1. Guía Conceptual. México, Inmujeres.

GG (2007). Glosario de Género, Noviembre, México, Inmujeres.

Inegi (2014). Instituto Nacional de Estadística, Geografía e Informática/Instituto Nacional de las Mujeres, "Mujeres y Hombres en México 2013".

Inegi (2015). Instituto Nacional de Estadística, Geografía e Informática/Instituto Nacional de las Mujeres, "Mujeres y Hombres en México 2014", México.

Inegi (2010). Instituto Nacional de Estadística y Geografía, "Resultados de la Encuesta Nacional de Gobierno, Seguridad Pública y Justicia Municipal 2009", Aguascalientes, Aguascalientes, México. 
Inegi (2007). Instituto Nacional de Estadística, Geografía e Informática/Instituto Nacional de las Mujeres, Mujeres y Hombres en México 2007.

LINM (2001). Ley del Instituto Nacional de las Mujeres, Diario Oficial de la Federación, México, 12 de enero.

LGAMVLV (2007). Ley General de Acceso de las Mujeres a una Vida Libre de Violencia, Diario Oficial de la Federación, México, $1^{\circ}$ de febrero.

LGIHM (2006). Ley General para la Igualdad entre Mujeres y Hombres, Diario Oficial de la Federación, México D.F., 2 de agosto.

PND (2007). Plan Nacional de Desarrollo 20072012, México.

PND (2013). Plan Nacional de Desarrollo 20132018. México.

PNIHM (2009).Programa Nacional para la Igualdad entre Mujeres y Hombres 2009-2012. Diario Oficial de la Federación. 18 de agosto de 2009. México.

PNIOND (2013). Programa Nacional para la Igualdad de Oportunidades y no Discriminación contra las Mujeres 2013-2018. Diario Oficial de la Federación. 30 de agosto. México. 


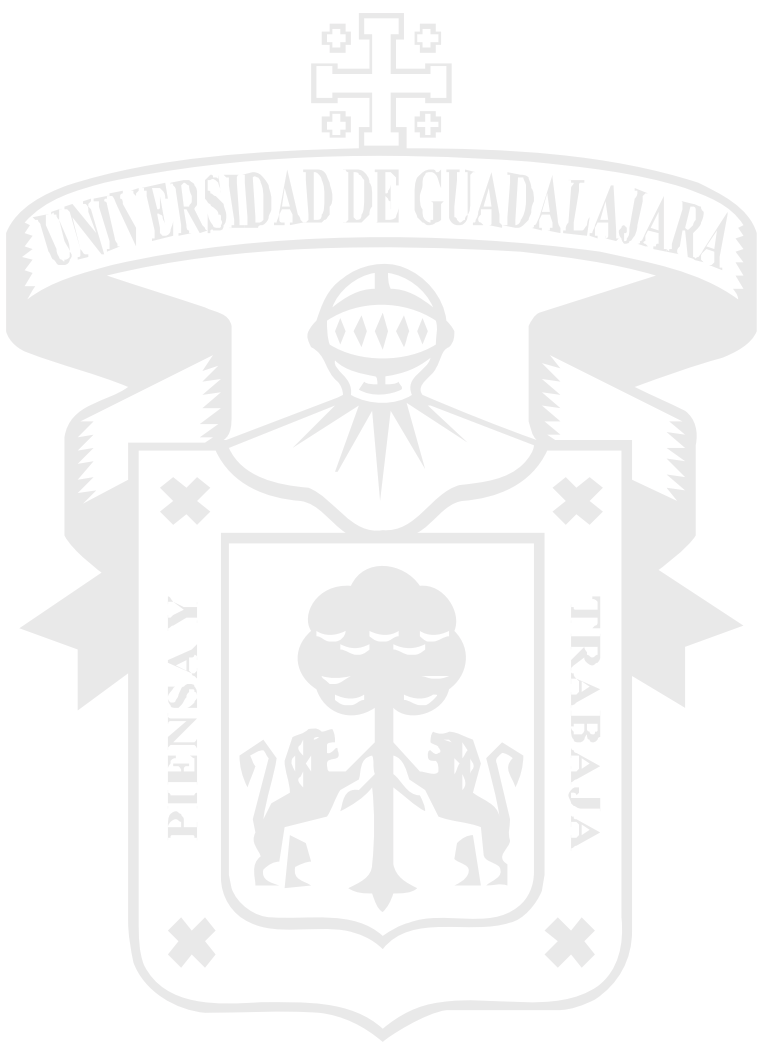

